Chapter forthcoming in: Gesture in language: Development across the lifespan, edited by A. Morgenstern \& S. Goldin-Meadow

\title{
Pointing in gesture and sign
}

\author{
Kensy Cooperrider ${ }^{1}$ \\ Kate $\mathrm{Mesh}^{2}$ \\ ${ }^{1}$ University of Chicago, Department of Psychology \\ ${ }^{2}$ Lund University, Lund University Humanities Lab
}

\section{Introduction}

Human communication is composite (e.g., Clark 2016; Enfield 2009; Ferrara and Hodge 2018; Holler and Levinson 2019). It involves the voice, face, hands, and rest of the body. It integrates categorical elements and gradient ones; highly conventional and ad hoc forms; arbitrary symbols and motivated signals. This is true of spoken communication and it is true-in equal measure - of signed communication. Both speakers and signers stitch these different types of components into a seamless whole. Some of these components are historically considered a core part of language, others marginal, and still others are thought to be something else entirelygestural, expressive, paralinguistic (for discussion, see, e.g., Dingemanse 2018; Goldin-Meadow and Brentari 2017; Müller 2018). Regardless of whether one considers the language/ non-language divide fundamental, fuzzy, or fictitious, there is widespread agreement that certain communicative phenomena haunt the boundaries in ways that prove revealing. Chief among these are cases where both speakers and signers make use of the same bodily raw material, but in putatively very different ways: flashes and furrows of the brow; imitations of actions; depictions of size, shape, and arrangement. Here, we analyze one of these similar-looking forms in detail: pointing. The case of pointing shows, first, how a single semiotic tool can be put to many uses and, second, how speakers and signers use this tool in some ways that are similar and other ways that are different.

Pointing is an especially powerful and pervasive tool in the semiotic kit, used across the lifespan, across cultures, and across contexts. It's a major way that humans coordinate attention,

\footnotetext{
* Note: This is the accepted version of a chapter in press; it does not include minor changes that may result from copy-editing. Please quote only the final published version.
} 
anchor words to the world, and build common ground with each other. Following others, we will here define pointing as a bodily "movement toward" a target - someone, something, somewherewith the intention of reorienting attention to it (Eco 1976; see also Cooperrider, Slotta, and Núñez 2018; Kendon 2004). Often this gesture is done with the index finger - a pre-eminently "pointy" articulator that projects an imaginary vector-but it can also be done by tossing the head, pursing the lips, or extending a machete, among other ways. Pointing is a means of indicating - that is, of establishing attention to something by creating a spatiotemporal connection to it (Peirce 1940; see also Clark 2003). It is not the only way of indicating — one can also pat something or hold it up for inspection (Clark 2003). Indicating, in turn, is one of the three major methods of meaning-making that humans have-along with depicting (that is, using iconic representations) and what is sometimes called describing (that is, using symbolic resources) (Clark 2003, 2016; Enfield 2009; Ferrara and Hodge 2018; Peirce 1940). (The term "symbolic" is used in many ways in linguistics and cognitive science, but here refers to meaning conveyed by rule or convention, e.g., that a green traffic light means 'go.') On purely theoretical grounds, then, pointing is a "basic building block" of communication (Kita 2003a). And so it is on empirical grounds, too. Pointing is an earlyemerging communicative act — among the earliest, in fact (see Morgenstern, this volume) — and it is found universally in both spoken and signed communication (Kendon 2010; Kita 2003a; Morgenstern 2014; Pfau 2011).

Unsurprisingly, this elemental gesture has attracted the attention of both gesture researchers and sign language linguists. However, scholars in these two traditions have looked at pointing through different lenses and have gravitated toward different aspects of it. Gesture researchers, for example, have usually treated pointing as an adjunct to language but not really part of it; sign researchers, in contrast, have often treated pointing as a core part of sign language grammar rather than as a separate, "gestural" component (e.g., Meier and Lillo-Martin 2010). These differing frameworks and foci contribute to an impression that-superficial similarities notwithstanding - pointing gestures and pointing signs are, deep down, fundamentally different. Recently, however, there is a new push to compare pointing gestures and pointing signs directly, that is, using similar datasets and similar analytical criteria. These direct comparisons underscore the fact that pointing gestures and pointing signs share many commonalities, and help sharpen our understanding of where exactly the differences lie. 
Pointing may be a basic, foundational communicative tool, but - as we emphasize hereit is also a multifarious one. We thus examine pointing in all its formational, functional, contextual, and cultural variety. In what follows, we begin by looking closely at the major foci of research on pointing in gesture studies $(\S 2)$ and in sign language linguistics $(\S 3)$. We then review recent efforts to directly compare the two $(\S 4)$. A major refrain throughout will be that, contrary to its assumed simplicity, pointing is multiform and multifunctional in both gesture and sign.

\section{Pointing gestures}

Though there is a rich tradition of research on pointing in children (e.g., Bates 1976; Cochet and Vauclair 2010; Tomasello, Carpenter, and Liszkowski, 2007; see Morgenstern, this volume), research on adult pointing gestures has been more sporadic and diffuse. It has originated from diverse disciplinary quarters - including anthropology, psychology, linguistics, and conversation analysis. Despite this diversity of approaches, these efforts have had a few recurring foci, including: the variety of uses of pointing, with some uses considered "primary" and others "secondary"; the relationship of pointing to spoken language; how pointing varies in form from one use to the next; and how pointing varies across cultures. We now consider these foci in turn.

\subsection{Primary and secondary functions of pointing}

One focus of research in gesture studies has been the variety of functions pointing serves in communication. By definition, pointing always serves the function of drawing attention somewhere. But, under this broad umbrella, pointing has certain uses that are widely considered “primary” (Enfield, Kita, and de Ruiter 2007), "prototypical” (Langacker 2008), or "canonical” (Cooperrider 2014), and others that are usually considered "secondary". The primary use of pointing, on these treatments, involves indicating something in the real world — such as a star in the sky, a mountain on the horizon, a fish in an aquarium — and, in doing so, inviting a listener to look at that something. There are many contexts in which such points occur, including ostensionbased language learning (e.g., Clark and Estigarribia 2011), direction-giving (e.g., Kita 2003b),

\footnotetext{
1 "Primary" and "secondary" are, of course, theoretically loaded terms, inviting the question: Primary in what sense? One idea is that primary points are more frequent - as far as we know, there is no work suggesting this. Another idea is that they loom larger in folk theories of pointing - this has been claimed, but without any direct evidence. A third idea is they are learned first. This seems likely to be the case, but, again, we are not aware of direct evidence.
} 
sight-seeing (e.g., Kendon 2004), museum visits (Dimitra 2012), and a variety of other joint activities (e.g., Bangerter 2004). By definition, primary points not only invite listeners to re-orient their gaze, they also convey crucial information about where something is, or which of several is meant (Enfield, Kita, and de Ruiter 2007). Without the information conveyed by such gestures, the communicative message would be incomplete.

But pointing is also used in a number of other ways that are often considered "secondary," even within the category of "real-world" points to entities or places (see Figure 1). One example is when people point to something or somewhere, but without necessarily intending to redirect listener gaze and without relying on the point to communicate message-critical information. Enfield, Kita, and de Ruiter (2007) describe pointing in such cases as a kind of pragmatic safety net; it is used when the speaker thinks the listener knows the referent but is not entirely sure. Relatedly, speakers point in cases where the listener is already attending to the pointed-to target and where the referent is perfectly clear. A good example is seen in points to the self (Cooperrider 2014). When speakers point to their own bodies along with pronouns like 'I,' 'my,' or 'mine,' they are drawing attention but not necessarily reorienting listener gaze-according to the norms of conversation, listeners should already be looking at the speaker, and the referent of ' $\mathrm{I}$ ' is rarely ambiguous. Similarly, when pointing to the listener with 'you' or 'yours,' listeners know where they are and the referent is usually not ambiguous. In these cases, pointing serves to reorient discourse attention but not visual attention per se; it adds emphasis but does not contribute message-critical information.

Another type of "secondary" pointing occurs when people point to one thing to refer to another. In the above examples, what the speaker points to - the target - is recognizably the same as what is referred to in speech — the referent. This is sometimes called "direct pointing" (Le Guen 2011). But, at other times, the pointed-to target is associated with the referent but not identical to it (e.g., Borg 2002; Clark 2003; Le Guen 2011). This phenomenon has gone by different labels, including "metonymic pointing," "deferred ostension," and "indexical chaining." A classic example involves pointing to a speedometer to refer to a car's speed (Quine 1960); other examples include pointing to the chest to refer to a 'we' (Cooperrider 2014), or pointing to a house to refer to one of its occupants (Levinson 2006). 


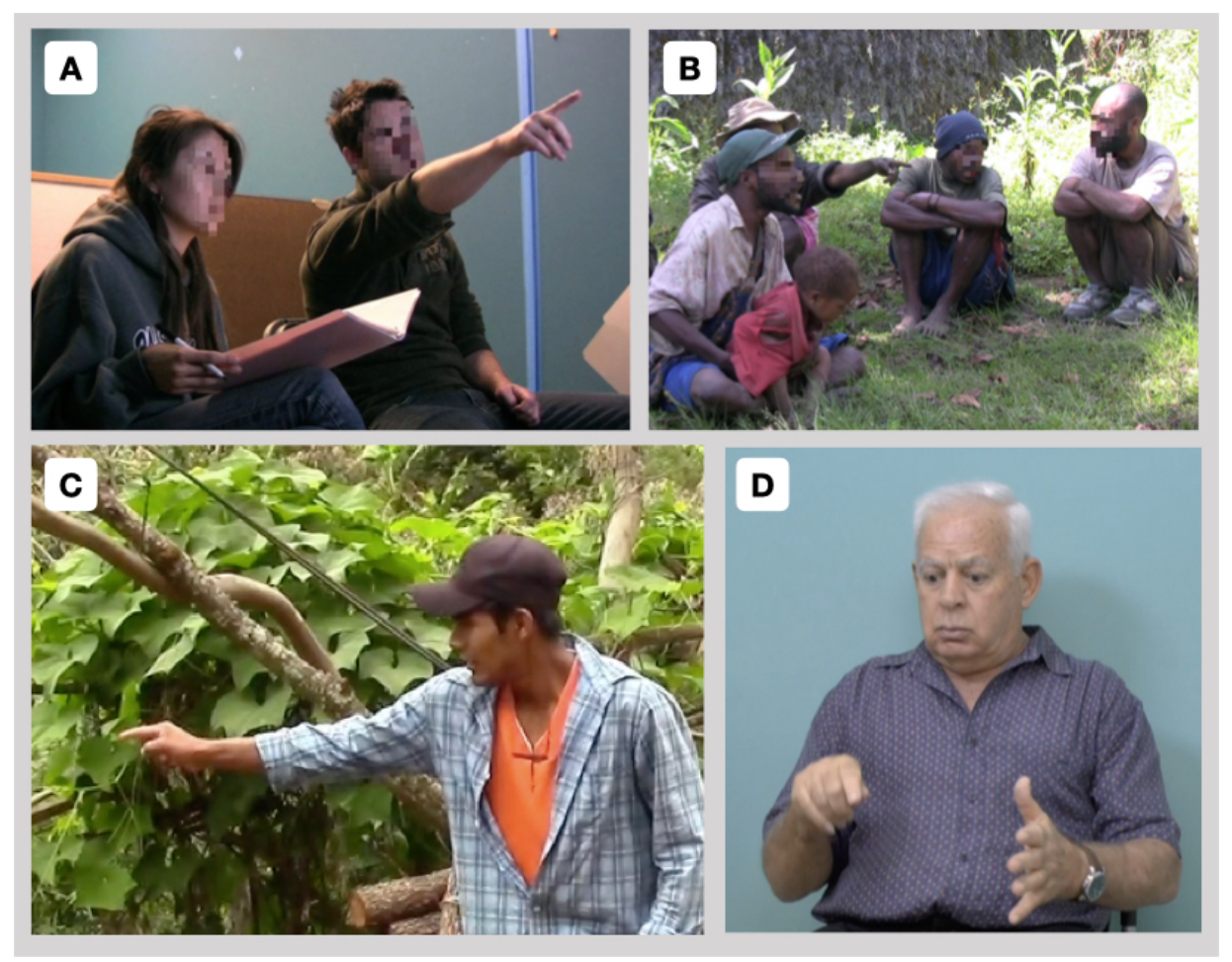

Figure 1. Examples of points to real-world entities in gesture (top row) and sign (bottom row). (A) An English speaker points to an array of novel creatures, while carrying out a referential communication task. (B) A Yupno (Papua New Guinea) man, far right, asks a "where" question, and his three interlocutors point as part of their answers: a nose point (far left), an index finger point (man behind, face occluded), and another nose point (middle). (C) A deaf signer of San Juan Quiahije Chatino Sign Language (Mexico) points to a plant while he explains its various uses. (D) A deaf signer of Israeli Sign Language points to a foam block on the table in front of him while carrying out a referential communication task ${ }^{2}$.

People also point to things that, strictly speaking, are not there. This phenomenon is commonly known as deixis am phantasma (Bühler [1934] 1990) or abstract deixis (Stukenbrock 2014) and it takes a number of different forms. In some cases, people point metaphorically, such as to a temporal landmark like 'tomorrow,' which has no physical location in space (Cooperrider, Núñez, and Sweetser 2014). In other cases, people point to empty locations to invest them with

\footnotetext{
${ }^{2}$ We thank Wendy Sandler and the Sign Language Research Lab at the University of Haifa for granting permission to use this image.
} 
meaning (see Haviland 2000 on "baptismal" pointing), a behavior that has been studied in storytelling situations (McNeill 1992) and in joint activities (Bavelas and Gerwing 2011). This general technique of assigning referents to empty locations in space has been the subject of direct comparisons between speakers and signers, as discussed later. Finally, people also point to apparently empty space when they are gesturing "under transposition." That is, during storytelling, people may point as if from some imagined there-then rather than from the actual location herenow of the speech event (Haviland 1993, 2003).

All of the uses of pointing considered so far serve referential functions - they serve to draw attention to a person, place, object, or idea being overtly referred to in the discourse. But points sometimes also serve more narrowly interactive functions. This often involves pointing to present people. For instance, speakers taking over a turn may point to the last speaker as a way of showing agreement with what they just said, even though that speaker goes unmentioned in the discourse (Healy 2012). Similarly, in multiparty conversations such as meetings, people point as a way of tacitly citing others present (Bavelas et al. 1992). Pointing to the addressee is also used, not to show agreement, but to mock (Sherzer 1973) or scold (Andrén 2014). Generally, such social functions of pointing have not been as widely examined as the more prototypical referential uses. Note, of course, that these interactive functions still involve the same overarching function of orienting attention to a region of space - in the case the person being agreed with, cited, mocked, or scolded - but take on a richer meaning in context. Moreover, even a point that is prototypically referential—such as a point to someone while addressing them-may do important social work, as when it conveys authority or reprimand.

\subsection{Co-production with speech}

Another focus for gesture researchers has been how pointing is organized in relation to spoken language. Importantly, pointing does sometimes occur on its own, without accompanying speech - early in development but also in adult communication. Generally, like depicting gestures, points can occur on their own, in sequence with speech, or overlapping with speech (Clark 2016). When pointing does overlap with speech, it is most prototypically associated with a distinctive class of words known as demonstratives — including, in English, this, that, these, those, here, and there (Diessel 2006). Indeed, demonstratives have sometimes been dubbed "pointing words" (Diessel 2012). This is partly because demonstratives commonly co-occur with pointing-some 
describe pointing as obligatory when demonstratives are used (e.g., Levelt, Richardson, and Heij 1985) - and partly because both serve to indicate something in the world. Going further, Cooperrider (2016) has emphasized that demonstratives and pointing are designed in relation to each other, or "co-organized." In particular, the choice of whether to point to an entity is entwined with the choice of whether to use a demonstrative and, if so, whether to use this or that, here or there (or their parallels in other languages, see Mesh et al. in press; Piwek, Beun, and Cremers 2008).

Pointing also commonly co-occurs with spoken language beyond demonstratives, of course. Because points are often used for conveying "where" or "which" information, pointing is regularly used along with location or feature descriptions (Bangerter 2004). In fact, the range of spoken referents that pointing can partner with is essentially unbounded. By making use of metonymy, metaphor, and imagination, speakers can talk about a wide world of possible referents-non-present, invisible, non-physical—while simultaneously directing attention to regions of space in the here-and-now (Cooperrider 2014).

\subsection{Variation in pointing across contexts}

Gesture researchers have also examined how points vary in form from use to use and context to context. Such variation is usually not assumed to be arbitrary, but rather to reflect finegrained differences in function. Some aspects of this variation stem from culture-specific conventions, as discussed later, but others may reflect general principles. For example, Kendon (2004) describes how different pointing handshapes are tailored to different discourse purposes. He notes that when British and Italian speakers indicate something for the purposes of presenting it for "inspection" (224) they tend to point with the palm open and facing up. In other cases, variation in pointing handshape reflects the incorporation of iconic features, thus fusing indicating and depicting elements (Cooperrider 2014; Goodwin 2007; Kendon 2004). Recently, Talmy (2018) has analyzed in detail such deviations from the prototypical case of index finger pointing, creating a typology of how different ways of pointing evoke targets that are static or moving, $2 \mathrm{~d}$ or 3d, punctate or extended (see also Hassemer and McCleary 2018).

Beyond incorporating iconic features, pointing gestures also vary from use to use in how much effort the speaker puts into them. Drawing on interviews with Lao speakers, Enfield, Kita, and de Ruiter (2007) observed that points serving the primary function of conveying location 
information involved greater arm extension and were more likely to involve speaker gaze to the target; the secondary points they observed, in contrast, were "smaller" in form. Relatedly, Bangerter and Chevalley (2007) observed that "communicative points"-produced when speaker and listener were visible to each other-were more likely to involve arm extension than "noncommunicative points" - produced when a barrier separated the participants. These and other findings suggest that pointing gestures embody varying degrees of effort. They also suggest a candidate general principle that merits further investigation: the more central a pointing gesture is to the message at hand, the more effort the speaker will put into it.

\subsection{Variation in pointing across cultures}

A final focus has been on how pointing varies from one culture to the next. Pointing, by all accounts, is a human universal (e.g., Cooperrider, Slotta, and Núñez 2018), but it varies in several ways across communities. Some of this variation is due to particular conventions of pointing form. Speakers of Arrernte, an Australian Aboriginal language, have several pointing handshapes that are codified for particular purposes - for instance, an open hand with palm facing to the side is used when indicating the direction of an absolutely oriented path (Wilkins 2003). Some communities have a conventional practice of raising the height of the pointing arm to reflect the distance of the target - the higher the arm, the farther away the target (e.g., Eco 1976). People in Mesoamerica show an especially exaggerated version of this "far-is-up" strategy, sometimes using a near-vertical point to indicate distant referents (Le Guen 2006; Levinson 2003; Mesh 2017, submitted). Different communities also have different conventions for pointing non-manually, with the head and face. Some form of pointing with the head — such as tossing, thrusting, tiltingappears to be universal (e.g., McClave 2007). In certain cultures, however, there are also conventional facial pointing actions. These include lip-pointing, which consists of protruding, funneling, or pursing the lips (Enfield 2001; Mihas 2017; Sherzer 1973), and nose-pointing, which consists of scrunching the nose (Cooperrider and Núñez 2012). Both of these types of facial points are usually accompanied by a meaningful shift of gaze in the direction of the target (Adone and Maypilama 2014; Enfield 2001). Ethnographers have frequently claimed that such facial gestures are a major - or even preferred - form of pointing in the communities where they are used (e.g., Sherzer 1983). In one case, this claim has been borne out quantitatively. Using a referential communication task, Cooperrider, Slotta, and Núñez (2018) found that people in the Yupno valley 
of Papua New Guinea, where nose-pointing is used, were just as likely to point non-manually as manually.

Pointing also varies across cultures by virtue of being bound up with broader communicative practices and cognitive patterns. For example, Blythe, Mardigan, Perdjert, and Stoakes (2016) have described how pointing becomes an especially critical communicative resource in Murrinhpatha conversation because of cultural taboos on naming certain people and the places associated with those people. Elsewhere, pointing is recruited into a conventional practice for referring to the time of day. The best-studied case is found in the Brazilian Amazon (Floyd 2016). Nheenghatú speakers will point to an accurately oriented arc of the sun, running east to west, in order to refer to particular times (e.g., noon, by pointing directly overhead) or to more extended intervals (e.g., all afternoon, by sweeping a hand over the corresponding segment of the arc). Similar practices are found much more widely in speaking communities (see also Le Guen and Pool Balam 2012), as well as in some village sign languages (de Vos 2014). Finally, it is reported that people in some indigenous communities remain absolutely oriented and maintain accurate cognitive maps as they move through the world (Levinson 2003). There is thus a cultural expectation in such groups that people will point accurately, even to distant, unseen locations (Haviland 1993; Le Guen 2011). In Western cultural groups, there appears to be no such expectation; Americans, for instance, sometimes point with comic inaccuracy, even to familiar locations (e.g., Schegloff 1984).

A final source of variation across cultures is taboos that regulate how you can point or what you can point to. In Ghana, for instance, pointing with the left hand is considered impolite, and this prohibition has consequences for direction-giving (Kita and Essegbey 2001). In Aboriginal Australia, where avoidance registers are used during certain social interactions, speakers will often point in a more "constrained" fashion by using a fist or the elbow (Green 2019; see also Adone and Maypilama 2014). Elsewhere, taboos govern what you can point to. Famously, in some cultures, it is unacceptable to point to rainbows (Lee and Fraser 2001); in many Western cultures, it is considered rude to point to people, though this norm is unevenly observed and commonly violated (e.g., Jarmołowicz-Nowikow 2015).

\section{Pointing signs}


Signers, like speakers, point prodigiously. Every sign language documented thus farwhether used by a deaf child without access to a sign language model (i.e., a homesigner), a group of deaf people in an urban or village setting, or even by hearing people as an alternative to speechrelies heavily on pointing to serve multiple functions. Despite this fact, research on sign languages has historically focused on only a small subset of the many functions of pointing signs. When sign linguistics arose as a field of study in the 1960s, its practitioners were intent on demonstrating that sign languages are not merely elaborate gestural systems, but instead exhibit the same structures found in spoken language (see, e.g., Klima and Bellugi 1979). As a result, early research on pointing signs focused on those features that could be directly compared with speech, and sidelined pointing features with analogues in gesture. Only relatively recently has a welcome sea-change begun: more and more, sign linguists are attending to the full set of features of pointing signs, taking interest in the many features that are shared with pointing gestures. The major foci of research on pointing signs include: similarities between pointing signs and spoken pronouns, demonstratives, and locative expressions; uses of pointing signs to establish and maintain reference; other uses of pointing, some analogous to "secondary" pointing gestures; and finally, cross-linguistic comparisons of pointing signs. We now discuss each in turn.

\subsection{Pronouns, demonstratives and locatives: analogues to pointing signs?}

Signers, of course, point toward the objects, spaces, and people around them: pointing is as fundamental to their communication as it is for speakers. However, the push to compare sign with speech led sign language linguists to largely focus on just one type of real-world pointing: points toward present people. These were compared systematically with pronouns, the most basic resource for referring to persons in speech. There was a rich set of comparisons to be made, first in terms of function: both pointing signs and pronouns refer, that is, they identify speech act participants and track reference to those participants throughout the discourse (e.g., EngbergPedersen 1993; Liddell 1996; Lillo-Martin and Klima1990; Meier 1990; Pettito 1987; Senghas and Coppola 2001; van Hoek 1992). In addition, signed points to persons can take different forms based on whether the target is the signer, addressee, or a third, present person - and whether that target is singular or plural - a fact that many sign linguists take as evidence for the grammatical person- and number-marking that is found on pronouns (e.g., Meier and Lillo-Martin 2013). Moreover, person-referring pointing signs are subject to the same principles that determine the 
placement of pronouns in spoken languages, including the so-called binding conditions on anaphora (for discussion, see Meier and Lillo-Martin, 2010). In accounting for this evidence, sign linguists have disagreed, sometimes quite contentiously, about whether person-referring pointing signs are true pronouns, or can even be called linguistic. At the heart of the argument is the question of whether a language's lexicon needs to contain a finite, listable set of forms. Some authors claim that because some features of pointing are gradient — in particular, the direction of the point, which may be modified in indefinitely many ways - signed points should be understood as gestural components of the language (meaning, formed at least partly from gradient features) rather than linguistic (meaning, organized around a finite set of categorical oppositions) (e.g., Liddell and Metzger 1998; Liddell 2000; Liddell 2003). Other authors argue that pointing signs are organized in a way that makes them linguistic, but the types of distinctions they encode are limited and are thus closer to a simplified demonstrative system than to a pronominal one (Ahlgren 1990; Koulidobrova and Lillo-Martin 2016; McBurney 2002). Still others argue that the person- and number-marking features seen in some sign language's person-referring points justifies treating them not only as linguistic, but as clear pronouns (see discussion in Cormier, Schembri, and Woll 2013). A growing trend in the discipline is to side-step the debate altogether and not worry as much about categorizing pointing signs as linguistic or gestural. Such treatments focus instead on identifying similarities and differences between pointing signs and pronouns or demonstratives on the one hand, and pointing signs and pointing gestures on the other (see discussions in Cormier, Schembri, and Woll 2013; Johnston 2013a, 2013b); or on the cognitive processes that account for how speakers and signers use points (see discussion in Wilcox and Occhino, 2016).

Of course, signers point not only toward people in the world around them, but also toward objects and locations. Pointing signs targeting objects have been described as demonstrative expressions (Koulidobrova and Lillo-Martin 2016; McBurney 2004), while pointing signs targeting locations have been called 'locatives' (e.g., de Vos 2013; Padden 1983; Shepard-Kegl 1985). Notably, signers appear to distinguish points toward locations from points toward people by modifying two formational features of points: palm orientation and handshape. Points toward locations are typically formed with the palm facing downwards, whereas points toward people are more often formed with the palm facing to the side; this observation has been made for a number of the world's sign languages (for a review, see Pfau 2011), including in a quantitative analysis of British Sign Language (BSL) (Fenlon et al. 2013). Studies of American Sign Language (ASL) and 
of BSL have also shown that points toward locations are produced more often with an index finger (Bayley, Lucas and Rose 2002; Fenlon et al. 2013). Notably, in the ASL and BSL studies signers were more consistent in how they formed points toward locations, and showed more variation in their points toward people. Fenlon and colleagues (2013) suggest that this result is due to different patterns of co-articulation with the surrounding signs - a possibility that underscores how closely pointing signs are prosodically integrated with the signs surrounding them.

\subsection{Pointing signs to establish and maintain reference}

Some of the most interesting features of pointing in sign language arise when the point is directed toward nothing at all. Signers sometimes "anchor" a referent in space by first naming the referent, and then pointing to a location in the empty space in front of them (Barberà and Zwets 2013). An ASL signer recounting a story about her pet, for example, could introduce the animal with the lexical sign DOG, preceded or followed by a point. The noun-accompanying point appears to share the function of spoken language determiners, and its presence and ordering relative to the noun provides information about whether the reference is definite (the dog) or indefinite ( $a \operatorname{dog}$ ) (MacLaughlin 1997; Zimmer and Patschke 1990). Crucially, this type of point toward empty space — with or without an accompanying noun — has a second function: it associates the referent with the selected empty space (often called a referential locus, or R-locus) making it possible to point toward this same space later to refer back to the same referent. The ASL signer from our example points alongside the sign DOG, and in so doing, associates the notion of the dog with a specific location in the space in front of her. It is thus possible for her to continue to point to this same location throughout her narrative, referring again and again to the dog as she narrates his adventures (see discussion in Perniss and Özyürek 2015, Cormier, Schembri, and Woll 2013). Once a signer has associated a referent to a given R-locus, they can use a variety of deictic mechanisms beyond the point to refer back to the referent. Many sign languages contain a specialized set of main verbs that are produced using movements to or from R-loci, conveying that the subject or object of the verb is the referent associated with that space (e.g., Padden 1983; see also Hou and Meier, 2018; Schembri, Cormier, and Fenlon 2018). In our ASL example, the signer might modulate the location and movement of the verb BITE, making the starting-place of the moving hand the dog's R-locus (and thus identifying the dog as the biter) or moving the hand toward the dog's R-locus (identifying the dog as the bitee). 'Spatial agreement' or 'spatial 
modulation' of the kind exemplified by the movement of the verb BITE in this example is dependent on the meaningful association of referents with empty space, and this association is most often established by an initial pointing act. In this way, a seemingly marginal function of points- to establish reference to non-present entities - becomes foundational for verb inflection processes in many sign languages.

\subsection{Other pointing phenomena in sign languages}

Across signing communities, points are also regularly used metonymically - that is, points toward real-world spaces are used for referents that are not in those spaces, but are conceptually related to them (see Table 1). This, of course, is analogous to the metonymic pointing gestures described earlier. In Yolngu Sign Language and Kata Kolok, languages used in small-scale communities where the location of everyone's home is common knowledge, a signed point toward a particular home refers to the person who lives in it (Bauer 2014; de Vos 2013). Among not only speakers of Yucatec Maya, but also signers of Yucatec Maya Sign Language, a point to the sky refers to the time of day when the sun is at that location (Le Guen and Pool Balam 2012; see also de Vos 2013). In young sign languages and more established ones alike, points to the hair, teeth and lips are regularly used to refer to the colors black, white, and red (de Vos 2011; Nonaka 2004; Woodward 1989; Zeshan and Sagara 2016). The human propensity toward developing metonymic reference is so great that even when homesigners get little exposure to metonymic pointing in gesture, they nevertheless develop it. Using this strategy substantially expands the communicative potential of pointing (Butcher, Mylander, and Goldin-Meadow 1991).

Sign languages also incorporate pointing into fully lexical signs. For example, in ASL and other sign languages, body parts terms are most often formed by a pointing movement toward the body part. Often these are not simply prototypical points with an index-finger extended, but involve different handshapes (e.g., open hand) or motion (e.g., reduplication) (Pyers 2006). Indeed, many lexical signs, while not obviously "pointy," are articulated in relation to parts of the bodysuch as the head, face, or abdomen - and thus motivated, in part, by metonymic indexicality (Cooperrider 2014; Kendon 1980). For instance, words related to cognition are often articulated near the head (Evans and Wilkins 2000; Kendon 1980); in contrast, words related to hunger may be articulated near the stomach, and words related to eating may be articulated near the mouth (Östling, Börstell, and Courtaux 2018). 
Table 1. Research on uses of pointing in gesture and sign

\begin{tabular}{|c|c|c|}
\hline & Gesture & Sign \\
\hline \multicolumn{3}{|c|}{ Direct points to real-world entities } \\
\hline Objects & $\begin{array}{l}\text { e.g., Bangerter } 2004 \text {; } \\
\text { Cooperrider } 2016\end{array}$ & $\begin{array}{l}\text { e.g., Koulidobrova and Lillo- } \\
\text { Martin 2016; McBurney } 2004\end{array}$ \\
\hline Locations & $\begin{array}{l}\text { e.g., Enfield, Kita, and de } \\
\text { Ruiter 2007; Mesh } 2017 \text {, } \\
\text { submitted; Wilkins } 2003\end{array}$ & $\begin{array}{l}\text { e.g., de Vos 2013; Padden } \\
\text { 1983; Shepard-Kegl 1985; }\end{array}$ \\
\hline Persons & $\begin{array}{l}\text { e.g., Cooperrider 2014; } \\
\text { Jarmołowicz-Nowikow } 2015\end{array}$ & $\begin{array}{l}\text { e.g., Cormier, Schembri, and } \\
\text { Woll 2013; Meier and Lillo- } \\
\text { Martin, 2010, } 2013\end{array}$ \\
\hline \multicolumn{3}{|l|}{ Metonymic points } \\
\hline $\begin{array}{l}\text { Locations for Person } \\
\text { reference }\end{array}$ & e.g., Levinson, 2006 & $\begin{array}{l}\text { e.g., Bauer 2014; Butcher, } \\
\text { Mylander, and Goldin- } \\
\text { Meadow 1991; de Vos } 2013\end{array}$ \\
\hline $\begin{array}{l}\text { Locations for Temporal } \\
\text { reference }\end{array}$ & $\begin{array}{l}\text { e.g., Floyd 2016; Le Guen } \\
\text { and Pool Balam } 2012\end{array}$ & $\begin{array}{l}\text { e.g., de Vos } 2013 \text {; Le Guen } \\
2012\end{array}$ \\
\hline $\begin{array}{l}\text { Body parts for Experiential } \\
\text { concepts }\end{array}$ & e.g., Cooperrider 2014 & $\begin{array}{l}\text { e.g., Evans and Wilkins 2000; } \\
\text { Kendon 1980; Östling, } \\
\text { Börstell, and Courtaux } 2018\end{array}$ \\
\hline Body parts for Colors & not attested & $\begin{array}{l}\text { e.g., de Vos 2011; Woodward } \\
\text { 1989; Zeshan and Sagara } \\
2016\end{array}$ \\
\hline \multicolumn{3}{|l|}{ Points to empty space } \\
\hline Referential Loci & $\begin{array}{l}\text { McNeill 1992; Perniss and } \\
\text { Özyürek } 2015\end{array}$ & $\begin{array}{l}\text { e.g., Cormier, Schembri, and } \\
\text { Woll 2013; Engberg-Pedersen } \\
\text { 1993; Liddell } 2003\end{array}$ \\
\hline Metaphorical & $\begin{array}{l}\text { e.g., Cooperrider, Núnéz, and } \\
\text { Sweetser } 2014\end{array}$ & $\begin{array}{l}\text { e.g., Yano and Matsuoka } \\
2018\end{array}$ \\
\hline Transposed targets & e.g., Haviland 1993 & e.g., Liddell 2003 \\
\hline
\end{tabular}




\begin{tabular}{|l|l|l|}
\hline$\underline{\text { Interactive functions of }}$ & $\begin{array}{l}\text { e.g., Bavelas et al. 1992; } \\
\text { pointing }\end{array}$ & e.g., Ferrara 2020 \\
\hline
\end{tabular}

\subsection{Pointing signs in crosslinguistic comparison}

When discussing the variety of functions for pointing signs, it can be easy to forget that the sign languages in which pointing is found are themselves remarkably diverse. There is no one context for "pointing in sign language": rather, pointing signs are found in sign languages young and old, in urban and rural environments, with high or low numbers of users in a variety of different social configurations. What is common to the pointing signs found in all of these environments is that they are frequent and indispensable. For homesigners still in the process of conventionalizing vocabularies, pointing is a reliable tool for identifying not only present objects but also the properties that they embody (Coppola and So 2006; Torigoe and Takei 2002). For signers of more established sign languages, pointing takes on additional functions (Pfau and Steinbach 2006) and in at least some contexts it is used even more frequently than in homesign (Coppola and Senghas 2010). There are certainly aspects of variation in pointing across sign languages. For example, pointing signs draw attention to the physical environment in ways that reflect the different topographies and direction-giving traditions where sign languages emerge (de Vos 2013; Mesh 2017, submitted; Nonaka 2015), and they direct attention beyond the here-and-now in ways particular to the narrative practices of specific cultures (Green and Wilkins 2014). Just how uniformly pointing is integrated into different sign languages, and how much diversity there may be in sign language pointing practices, are promising areas for further study.

\section{Comparing gesture and sign}

Much of the work on pointing gestures has been done without drawing any comparisons to pointing signs; and, vice-versa. Fortunately, this is starting to change. Increasingly, for instance, sign linguists are comparing phenomena in sign both to analogous phenomena in spoken language and to analogous phenomena in co-speech gesture (e.g., Cormier, Schembri, and Woll 2013; Johnston 2013a; Meier and Lillo-Martin 2013; Pfau 2011). A number of insightful observations have come out of such theoretical comparisons, and a range of similarities and differences between 
pointing gestures and pointing signs have been proposed. One limitation of such theoretical treatments, however, is that they often rely on an "armchair" understanding of pointing, rather than an empirically informed one. Moreover, because gesture researchers and sign researchers have so often gravitated to different aspects of pointing, it is tempting to conclude from the existing literature that gesturers don't really do this, or signers don't do much of that. But, in reality, we

simply lack studies spanning the full range of pointing behaviors in gesture and sign. To overcome this limitation, direct comparisons are critical (as some have noted, e.g., Cormier, Schembri, and Woll 2013). In this vein, several studies have, for instance, compared how signers and gesturers use pointing (and other forms of deictic anchoring) during reference tracking (Barberà and Zwets 2013; Perniss and Özyürek 2015). Here, we consider two recent lines of our own work; both are efforts to quantitatively compare pointing in gesture and sign using similar datasets and similar analytic criteria.

\subsection{Comparing pointing in Chatino gesture and sign}

A first study to systematically compare pointing by signers and gesturers in the same community, taking a quantitative approach to a sizable dataset, was performed by Mesh (2017) in a Chatino community of Mexico. This study compared points to "landscape-scale" referentsincluding schools, churches, and trade locations in the surrounding mountainous terrainproduced by gesturers and signers as they gave route directions.

The Chatino people traditionally inhabit a region at the base of the southern Sierra Madre mountain range in Mexican state of Oaxaca. While Chatinos are in no way socially isolated, there are social barriers to sending children to residential schools in mestizo (i.e., non-indigenous) cities. As a result, deaf Chatinos have minimal exposure to the national sign language transmitted in residential deaf schools. In the Chatino community of San Juan Quiahije, 13 of the approximately 3,600 community members are deaf, and these 13 people, along with their hearing family members, are developing an interrelated set of family sign languages: San Juan Quiahije Chatino Sign Language (SJQCSL). Signers of SJQCSL draw on the rich gestural practices found in the surrounding community. An interesting question that arises in this context is thus how much signers alter these practices as they incorporate them into a fully visual-manual language (Mesh and Hou 2018). Mesh (2017) approached this question by focusing on pointing practices in particular. 
In a series of semi-structured interviews, deaf and hearing citizens of San Juan Quiahije were asked how to reach local and regional landmarks on footpaths and via the roads that have more recently been built for travel by truck to distant locations. 31 people were interviewed: 29 hearing speakers of San Juan Quiahije Chatino (providing more than six hours of footage) and two deaf signers of SJQCSL, representing two distinct family sign varieties (providing 31 minutes of footage). Since pointing in Mesoamerica has been described as obeying the "far-is-up" principle (see §2.4), and as using different handshapes to indicate nearby, visible objects versus distant directions (e.g., Haviland 2003), all points were coded for the distance of the target (measured in meters) and for two formational features of the point: elbow height and handshape. A later extension of the study took a third formational feature into account: arm extension (Mesh submitted).

Chatino speakers showed a strong pattern of marking referent distance in their gestures, using all three coded features: they frequently indicated nearby targets using points with a low elbow, partial extension of the arm, and an extended index finger (Figure 2a). By contrast, they were significantly more likely to indicate distant targets using points with a high elbow, full extension of the arm, and an open handshape (Figure 2b). Remarkably, the two deaf SJQCSL signers mirrored the hearing speaker-gesturers' pointing system in only one respect: like gesturers, the signers used elbow height to mark distance, but unlike gesturers, the signers frequently used a fully extended arm and an extended index finger to indicate targets regardless of their distance (Figures $3 a$ and $3 b)$.

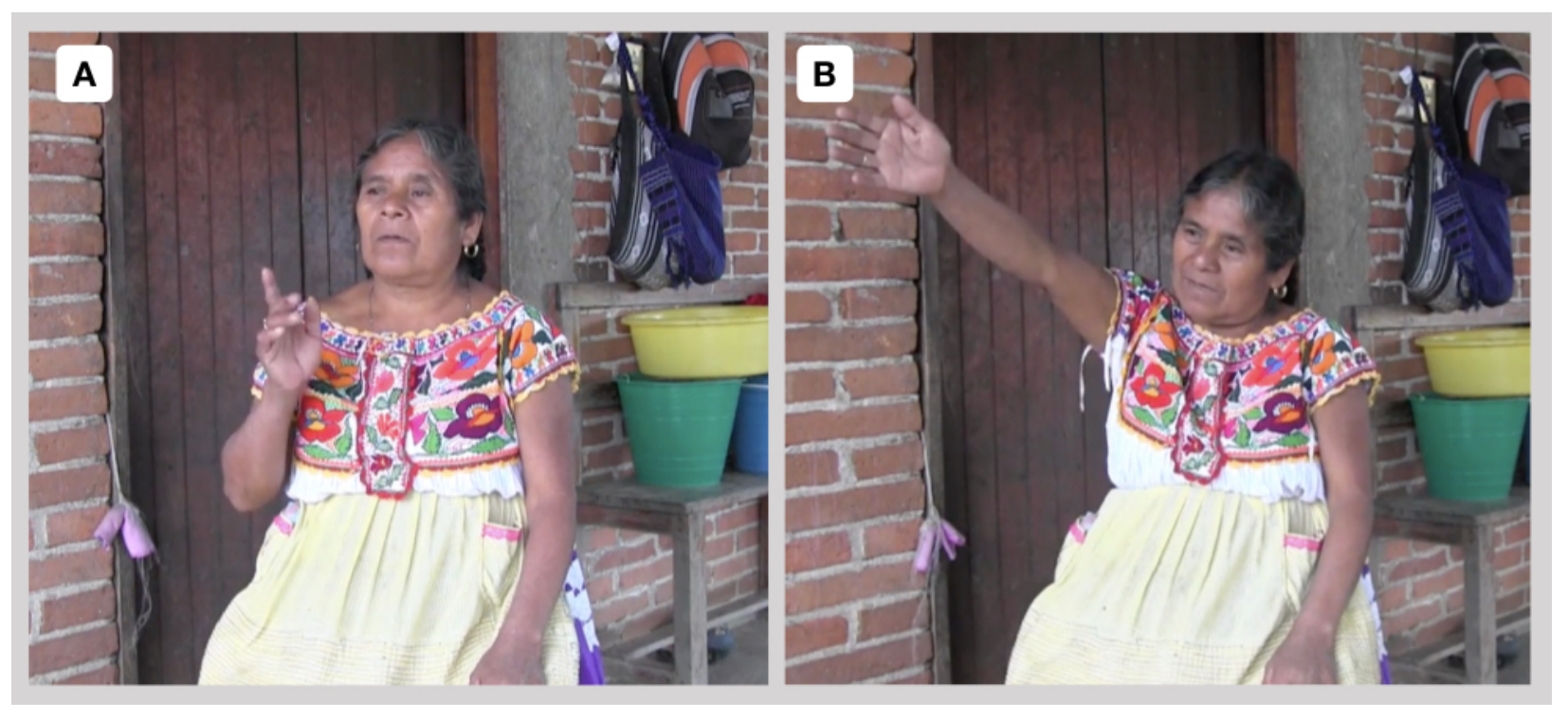


Figure 2. A speaker of San Juan Quiahije Chatino follows the "far is up" pointing principle: she points to a nearby street with a low, unextended arm and an outstretched index finger (A) and to a distant city using a high, extended arm with an open hand (B).

How can this result shed light on what is shared, and what is distinct, in pointing signs and pointing gestures? The area of similarity between gesturers and signers provides an important first clue: the use of elbow height to mark referent distance has been argued to be a universal feature of human pointing (see, e.g., Eco 1976), and even to extend to pointing in other species (Gonseth et al. 2017), but the instantiation of the far-is-up strategy varies across human communities. In particular, the use of near-vertical pointing to mark distant referents has been described as especially prominent in the Mesoamerican context (e.g., Levinson 2003). The pointing feature shared by signers and gesturers in San Juan Quiahije, then, is community-particular, and was evidently acquired in a process of cultural transmission involving both deaf and hearing recipients.

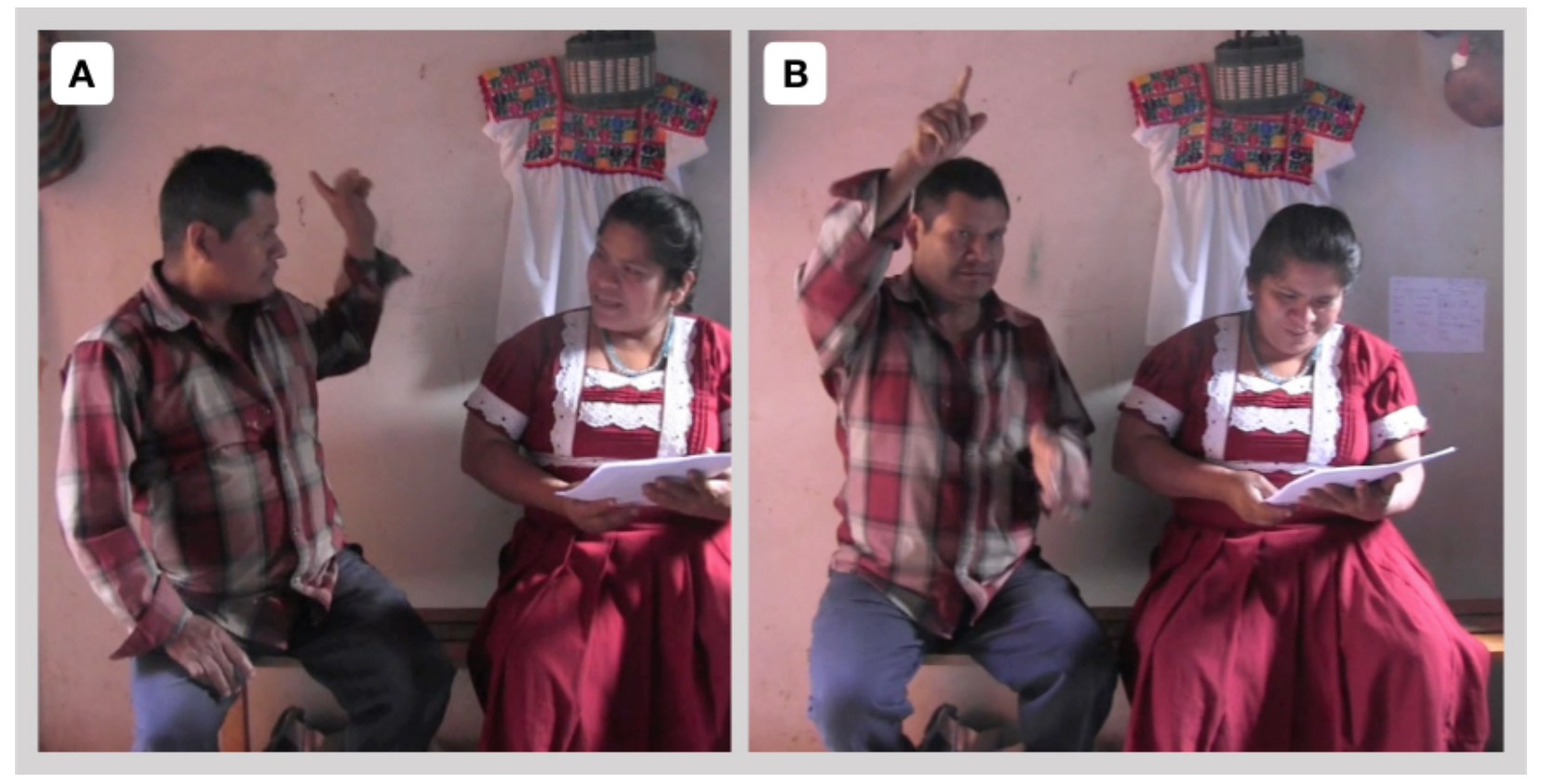

Figure 3. Signers of of San Juan Quiahije Chatino Sign Language also follow the "far is up" pointing principle: unlike speakers, though, they point with the same handshape for nearby targets (A) and distant ones (B). 
The differences between gesturers and signers in this study are equally important for our understanding of how pointing enters sign languages. Neither of the two family sign languages represented in this study had a pointing system identical to that of gesturers in the surrounding community. This gives us evidence that signers of emerging languages do not adopt the pointing practices around them wholesale. Rather, they differentially integrate features of pointing practices into their emerging linguistic systems, in ways that are likely sensitive to the contrasts already developing in their language's phonology and morphology. If differences are to be found between pointing gestures and pointing signs in communities with older, more established sign languages, these differences may well be due to language-specific constraints imposed on the adoption and adaptation of gestures during the early stages of the sign languages' emergence.

\subsection{Pointing in BSL, ASL, and spoken English}

Fenlon, Cooperrider, and colleagues recently compared pointing signs and pointing gestures using existing corpora, in a first study (Fenlon et al. 2019), and controlled elicitation, in a second (Cooperrider et al. 2021). The first study examined points to the self, addressee, and other entities (thus corresponding to first-, second-, and third-person pronouns) (Fenlon et al., 2019). The data came from two existing corpora of dyadic conversation; it included 27 English speakers from the Tavis Smiley Corpus (Cooperrider 2014), who contributed a total of 543 pointing gestures, and 24 signers from the conversational component of the British Sign Language Corpus (Fenlon et al. 2014), who contributed a total of 574 pointing signs. A number of prior researchers had suggested that pointing signs differ in their function from pointing gestures (e.g., Barberà and Zwets 2013; Meier and Lillo-Martin 2013). Fenlon and colleagues took a different tack, examining whether pointing signs might differ in their form from pointing gestures, by virtue of being more "linguistic" in nature. If so, the authors reasoned, the pointing signs should show a heightened degree of conventionalization, reduction, and prosodic integration, since these three characteristics are considered to be formational hallmarks of linguistic status.

By examining a range of features - such as handshape, hand use, duration, and others - the authors found that pointing signs did indeed differ from pointing gestures on these three dimensions. First, pointing signs appeared to be more conventionalized than pointing gestures. The signers were more consistent in their handshape preference, strongly favoring points with index finger extension; more consistent in using one hand instead of two; and more consistent in using 
their dominant hand. Second, pointing signs were much more reduced than pointing gestures, especially in terms of duration, lasting roughly a third as long as pointing gestures. Finally, the pointing signs were integrated into utterance-level prosody in a way that pointing gestures were not. Specifically, utterance-final pointing signs were longer in duration than non-final pointing signs - a pattern of lengthening widely observed for other types of signs (e.g., Wilbur 1999). Pointing gestures, in contrast, did not show this pattern.

These findings about form are thus consistent with the proposal that pointing signs are more linguistic than pointing gestures. However, the authors also noted an alternative possibility. Several of the observed differences might be explained instead by another crucial difference between sign and gesture: pointing signs are produced within the same articulatory channel as the rest of the referential content, i.e. the hands, whereas pointing gestures are produced in a different articulatory channel from the rest of the referential content. This "same channel" constraint offers an intuitive explanation for the shorter duration of pointing signs, as they have to be wedged into a stream of other signs. But the constraint could also have more subtle effects. For instance, it could add pressure to conserve effort when signing, leading signers to strongly favor one hand; it could also lead to pointing signs becoming more tightly integrated into broader prosodic structures because those structures are produced with the same articulators. In sum, it remains an open question whether the differences observed by Fenlon and colleagues are primarily driven by the linguistic status of pointing signs per se, or whether at least some might be driven by a samechannel constraint that exerts certain pressures on pointing signs.

A second study by the same researchers sought to further investigate commonalities and differences in pointing in gesture and sign, this time using controlled elicitation in the lab. The participants were 12 English speakers and 12 ASL signers (Cooperrider et al. 2021). Whereas the corpus study focused on pronoun-like points (to self, addressee, and other entities), the elicitation study focused on points to visible locations and objects. Of particular interest were two issues. A first was whether both pointing gestures and pointing signs would exhibit that pattern observed by Enfield, Kita, and de Ruiter (2007) for Lao speakers, in which "location-focus" points - that is, points carrying message-critical information about "which" or "where"-were bigger in form. To examine this, Cooperrider and colleagues designed a paradigm to elicit location-focus utterances in response to "which" or "where" questions (e.g., "That chair"), as well as explanatory utterances involving more than "which" or "where" information (e.g., "She walked to the chair in the back") 
(Figure 4). The expected pattern was that points embedded in location-focus utterances would be bigger in form than points embedded in explanatory utterances. Indeed, this pattern was found, but with an important additional wrinkle. The researchers further distinguished two types of points occurring within location-focus utterances: "load-bearing points" in which the point exclusively carried the locative information (e.g., "That chair"); and "load-sharing points" in which the point co-expressed the locative information alongside other locative words (e.g., "That chair on the right"). In both gesturers and signers, only the load-bearing points were larger in form; the loadsharing points were no bigger than those embedded in explanatory utterances.

A second issue was how the same-channel constraint described above might affect the integration of pointing signs with other signs (and, conversely, how the absence of this constraint might affect the integration of pointing gestures with spoken language). In line with the findings of Fenlon et al. (2019), pointing signs were markedly shorter in duration than pointing gestures. This was likely due to the contrasting ways in which pointing signs and pointing gestures were integrated into the surrounding linguistic materials. Pointing signs were far more likely to slot in between other signs (93\%) than they were to overlap with those signs (7\%) (i.e., by pointing with one hand while signing with the other); pointing gestures showed the opposite pattern, occasionally slotting in between spoken words (7\%) but far more often overlapping with them (93\%). Both signers and speakers thus make some use of both structural possibilities - slotting in and overlapping - but each group strongly favors one or the other. 


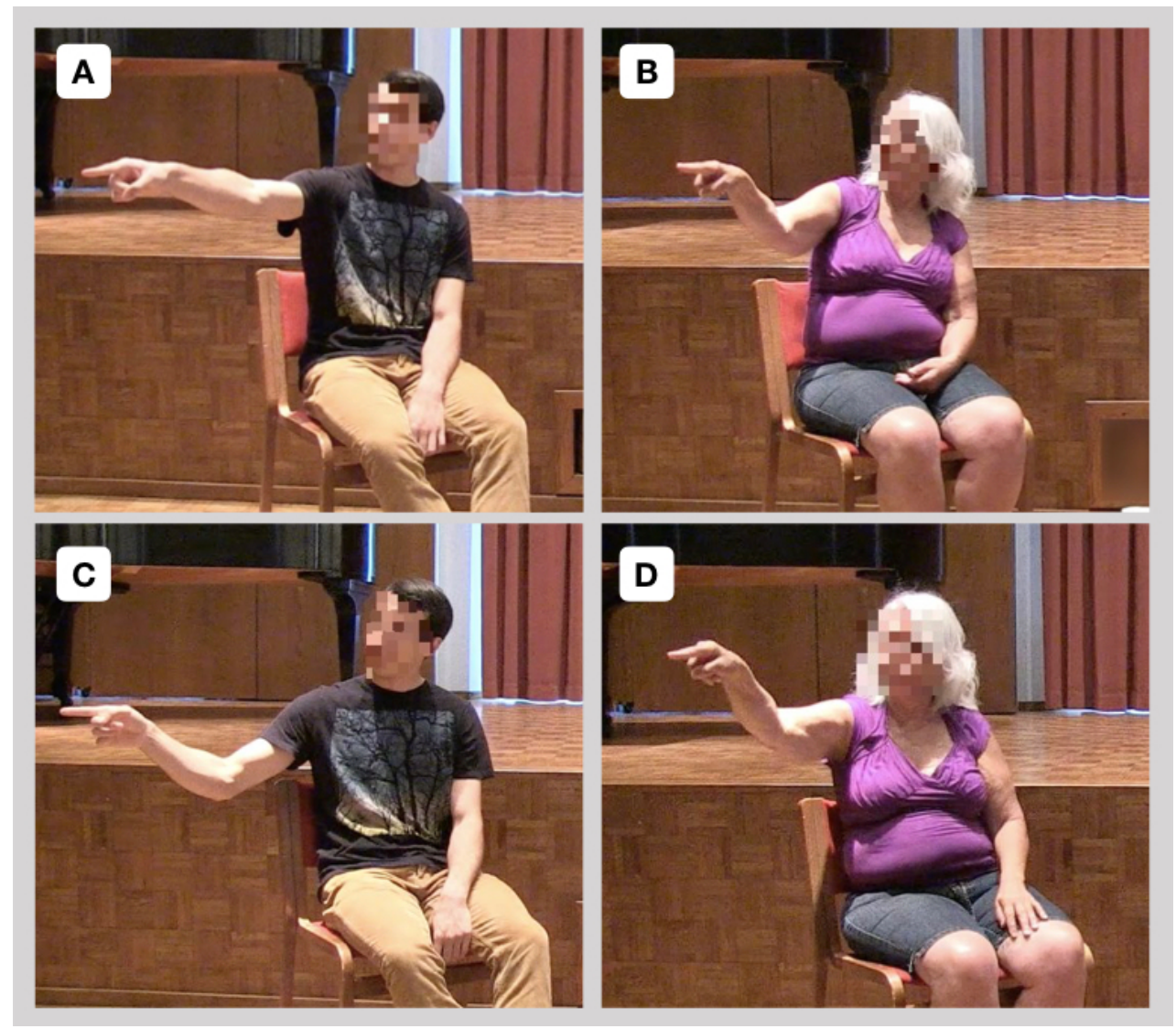

Figure 4. Examples of points to objects and locations produced by an English speaker (left column) and an ASL signer (right column). Points were produced as part of a referential communication task (Cooperrider et al. 2021), and were embedded in location-focus utterances (top row) or in explanatory utterances (bottom row).

Taking both studies together, several generalizations emerge. On the one hand, pointing gestures and pointing signs show a number of broad similarities. Both are used in similar ways, such as to point to present persons, non-present others, visible locations, and objects. Both are sometimes used along with other lexical material, and other times on their own. Both are responsive to similar functional pressures, such as the pressure to use more effort when the point carries central information. On the other hand, a number of broad differences were evident. Pointing signs are more consistent in form and tend to be more reduced, both in duration and in the bodily effort expended to produce them. This marked reduction may stem from a same-channel constraint that operates in sign but not in gesture. Of course, to corroborate these generalizations, more work is needed with different speaking and signing communities. 


\section{Conclusions}

Everyone points - children and adults, signers and speakers, urbanites and rural farmers. By any criterion we might choose-frequency of use, cross-cultural universality, developmental priority, semiotic simplicity-pointing is a basic communicative act. As such, pointing is sometimes treated as a monolith. But, in fact, pointing takes different forms and does different things; it varies from moment to moment and community to community; it has an over-arching function of directing attention, and a host of more fine-grained functions, too. It is integrated into spoken and signed communication in different ways, and this fact is sometimes reflected in its form. But this does not imply that pointing gestures and pointing signs are fundamentally, irreconcilably different. Nor does it imply that they sit on opposite sides of a language/ nonlanguage divide. As we have shown, many of the uses of pointing found in gesture - points to realworld people, objects, and places; metonymic points; points to empty space; points that serve interactive functions; and more - are also found in sign. Signers and speakers both point in a way that makes use of common conceptual mappings — such as the "far is up" mapping — and common pragmatic principles - such as the principle that more effort should be put into a point if it makes a critical contribution to the message.

Though we have sketched in broad strokes the similarities and differences between pointing gestures and pointing signs, it bears emphasis that there is much work left to do — and, in particular, there is much promise in further systematic, direct comparisons that will sharpen our understanding of these similarities and differences. As we continue to delve into other aspects of pointing-how it is formed, how it combines with gaze, how it is fitted into utterances, how it is deployed in particular interactive sequences - we will no doubt uncover more commonalities, as well as more points of divergence. Such direct, zoomed-in comparisons are not just critical for our understanding of pointing; they are critical for our understanding of human communication more broadly, of how speakers and signers make communicative wholes out of disparate parts.

\section{References}


Adone, Marie Carla D. \& Elaine Maypilama. 2014. Bimodal bilingualism in Arnhem land. Australian Aboriginal Studies 2. 101-106.

Ahlgren, Inger. 1990. Deictic pronouns in Swedish and Swedish Sign Language. In: Fischer, Susan D., \& Patricia Siple (eds.), Theoretical Issues in Sign Language Research 1. 167-174. University of Chicago Press.

Andrén, Mats. 2014. Multimodal constructions in children: is the headshake part of language? Gesture 14(2). 141-170. http://doi.org/10.1075/gest.14.2.02and

Bangerter, Adrian. 2004. Using pointing and describing to achieve joint focus of attention in dialogue. Psychological Science 15. 415-419.

Bangerter, Adrian \& Eric Chevalley. 2007. Pointing and describing in referential communication: When are pointing gestures used to communicate? In Van Der Sluis, Ielka, Mariët Theune, Ehud Reiter \& Emiel Krahmer (eds.), CTIT Proceedings of the Workshop on Multimodal Output Generation (MOG). Aberdeen, Scotland.

Barberà, Gemma \& Martine Zwets. 2013. Pointing and reference in sign language and spoken language: Anchoring vs. identifying. Sign Language Studies 13(4), 491-515.

Bates, Elizabeth. 1976. Language and context: The acquisition of pragmatics. Cambridge, MA: Academic Press.

Bauer, Anastasia. 2014. The use of signing space in a shared sign language of Australia 5. Berlin: Walter de Gruyter.

Bavelas, Janet B, Nicole Chovil, Douglas A. Lawrie \& Allan Wade. 1992. Interactive gestures. Discourse Processes 15. 469-489.

Bavelas, Janet \& Jennifer Gerwing. 2011. Dyadic evidence for grounding with abstract deictic gestures. In Stam, Gale \& Mika Ishino (eds.), Integrating gesture. 49-60. Amsterdam: John Benjamins.

Bayley, Robert, Ceil Lucas, \& Mary Rose. 2002. Phonological variation in American Sign Language: the case of 1 handshape. Language Variation and Change 14. 19-53.

Blythe, Joe, Kinngirri Carmelita Mardigan, Mawurt Ernest Perdjert, \& Hywel Stoakes. 2016. Pointing out directions in Murrinhpatha. Open Linguistics 2. 132-159. http://doi.org/10.1515/opli2016-0007

Borg, Emma. 2002. Pointing at Jack, talking about Jill: Understanding deferred uses of demonstratives and pronouns. Mind \& Language 17(5). 489-512. 
Bühler, Karl. 1990. Theory of language: The representational function of language. Philadelphia: John Benjamins.

Butcher, Cynthia, Carolyn Mylander \& Susan Goldin-Meadow. 1991. Displaced communication in a self-styled gesture system: Pointing at the nonpresent. Cognitive Development 6(3). 315-342.

Clark, Eve V. \& Bruno Estigarribia. 2011. Using speech and gesture to introduce new objects to young children. Gesture 11(1). 1-23. http://doi.org/10.1075/gest.11.1.01cla

Clark, Herbert H. 2003. Pointing and placing. In Kita, Sotaro (ed.), Pointing: Where language, culture, and cognition meet. 243-268. Mahwah, NJ: Lawrence Erlbaum.

Clark, Herbert H. 2016. Depicting as a Method of Communication. Psychological Review 123(3). 324-347. http://doi.org/10.1037/rev0000026

Cochet, Hélène, \& Jaques Vauclair. 2010. Features of spontaneous pointing gestures in toddlers. Gesture 10(1). 86-107.

Cooperrider, Kensy. 2016. The co-organization of demonstratives and pointing gestures. Discourse Processes 53(8). 632-656. http://doi.org/10.1080/0163853X.2015.1094280

Cooperrider, Kensy. 2014. Body-directed gestures: Pointing to the self and beyond. Journal of Pragmatics 71. 1-16. http://doi.org/10.1016/j.pragma.2014.07.003

Cooperrider, Kensy, \& Rafael Núñez. 2012. Nose-pointing: Notes on a facial gesture of Papua New Guinea. Gesture 12(2). 103-129. http://doi.org/10.1075/gest.12.2.01coo

Cooperrider, Kensy, James Slotta, \& Rafael Núñez. 2018. The preference for pointing with the hand is not universal. Cognitive Science, 42(4), 1375-1390.

http://doi.org/10.1111/cogs.12585

Cooperrider, Kensy, Rafael Núñez \& Eve Sweetser. 2014. The conceptualization of time in gesture. In C. Müller, A. Cienki, E. Fricke, S. Ladewig, D. McNeill, \& J. Bressem (Eds.), Bodylanguage-communication 2. 1781-1788. New York: Mouton de Gruyter.

Cooperrider, Kensy, Jordan Fenlon, Jon Keane, Diane Brentari, \& Susan Goldin-Meadow. 2021. How pointing is integrated into language: Evidence from speakers and signers. Frontiers in Communication. http://doi.org/ 10.3389/fcomm.2021.567774

Coppola, Marie \& Anne Senghas. 2010. Deixis in an emerging sign language. In Brentari, Diane (ed.), Sign languages: A Cambridge language survey. 543-69. Cambridge: Cambridge University Press 
Coppola, Marie, \& Wing Chee So. 2006. The seeds of spatial grammar: Spatial modulation and coreference in homesigning and hearing adults. In Bamman, David, Tatiana Magnitskaia \& Colleen Zaller (eds.), BUCLD 30: Proceedings of the 30th Annual Boston University Conference on Language Development. 119-130. Somerville, MA: Cascadilla Press.

Cormier, Kearsy, Adam Schembri, \& Bencie Woll. 2013. Pronouns and pointing in sign languages. Lingua 137. 230-247. https://doi.org/10.1016/j.lingua.2013.09.010

de Vos, Connie. 2011. Kata Kolok color terms and the emergence of lexical signs in rural signing communities. The Senses and Society 6(1). 68-76.

de Vos, Connie. 2013. Sign-Spatiality in Kata Kolok: How a village sign language of Bali inscribes its signing space. Nijmegen: Radboud University Nijemgen doctoral dissertation.

de Vos, Connie. (2014). The Kata Kolok pointing system: Morphemization and syntactic integration. Topics in Cognitive Science, 1-19. http://doi.org/10.1111/tops.12124

Diessel, Holger. 2006. Demonstratives, joint attention, and the emergence of grammar. Cognitive Linguistics 17(4). 463-489. DOI: 10.1515/COG.2006.015.

Diessel, Holger. 2012. Bühler's two-field theory of pointing and naming and the deictic origins of grammatical morphemes. In Breban, Tina, Lieselotte Brems, Kristin Davidse, \& Tanja Mortelmans (eds.), New Perspectives on grammaticalization: Theoretical understanding and empirical description. 35-48. Amsterdam: John Benjamins.

Dimitra, Christidou. 2012. Does "pointing at" museum exhibits make a point? A study of visitors' performances in three museums for the use of reference as a means of initiating and prompting meaning-making. London: University College London doctoral dissertation.

Dingemanse, Mark. 2018. Redrawing the margins of language: Lessons from research on ideophones. Glossa: A Journal of General Linguistics $3(1) .4$. DOI: http://doi.org/10.5334/gjgl.444

Eco, Umberto. 1976. A theory of semiotics. Bloomington, IN: Indiana University Press.

Enfield, Nicholas J. 2001. 'Lip-pointing': A discussion of form and function with reference to data from Laos. Gesture 1(2). 185-211. http://doi.org/10.1075/gest.1.2.06enf

Enfield, Nicholas J. 2009. The anatomy of meaning. Cambridge: Cambridge University Press.

Enfield, Nicholas J., Sotaro Kita, \& Jan-Peter de Ruiter. 2007. Primary and secondary pragmatic functions of pointing gestures. Journal of Pragmatics 39(10). 1722-1741. 
Engberg-Pedersen, Elisabeth. 1993. Space in Danish Sign Language: The semantics and morphosyntax of the use of space in a visual language. Hamburg: SIGNUM-Press.

Evans, Nicholas \& David Wilkins. 2000. In the mind's ear: The semantic extensions of perception verbs in Australian languages. Language 76(3). 546-592.

Fenlon, Jordan, Adam Schembri, Ramas Rentelis, \& Kearsy Cormier. 2013. Variation in handshape and orientation in British Sign Language: The case of the ' 1 ' hand configuration. Language \& Communication 33(1). 69-91.

Fenlon, Jordan, Adam Schembri, Ramas Rentelis, David Vinson, \& Kearsy Cormier. 2014. Using conversational data to determine lexical frequency in British Sign Language: The influence of text type. Lingua 143. 187-202. http://doi.org/10.1016/j.lingua.2014.02.003

Fenlon, Jordan, Kensy Cooperrider, John Keane, Diane Brentari, \& Susan Goldin-Meadow. 2019. Comparing sign language and gesture: Insights from pointing. Glossa 4(1). 2. http://doi.org/10.5334/gjgl.499

Ferrara, Lindsay. 2020. Some interactional functions of finger pointing in signed language conversations. Glossa 5(1). 88. DOI: http://doi.org/10.5334/gjg1.993

Ferrara, Lindsay \& Gabrielle Hodge. 2018. Language as description, indication, and depiction. Frontiers in Psychology 9. DOI: https://doi.org/10.3389/fpsyg.2018.00716

Floyd, Simeon. 2016. Modally hybrid grammar? Celestial pointing for time-of-day reference in Nheengatú. Language 92(1). 31-64.

Goldin-Meadow, Susan \& Diane Brentari. 2017. Gesture, sign, and language: The coming of age of sign language and gesture studies. Cambridge Core in Behavioral and Brain Sciences 40(e74). DOI: 10.1017/S0140525X1600039X

Goodwin, Charles. 2007. Environmentally coupled gestures. In Duncan, Susan, Cassell \& Elena Levy (eds.), Gesture and the dynamic dimensions of language. 195-212. Philadelphia: John Benjamins.

Gonseth, Chole, Fumito Kawakami, Etsuko Ichino \& Masaki Tomonaga. 2017. The higher the farther: Distance-specific referential gestures in chimpanzees (Pan troglodytes). Biology Letters 13(11). https://doi.org/10.1098/rsbl.2017.0398

Green, Jennifer. (Submitted). Embodiment and degrees of respect in speech and action.

Green, Jennifer \& David P. Wilkins. 2014. With or without speech: Arandic sign language from central Australia. Australian Journal of Linguistics 34(2). 234-261. 
Hassemer, Julius \& Leland McCleary. 2018. The multidimensionality of pointing. Gesture 17(3). 417-463.

Haviland, John B. 1993. Anchoring, iconicity, and orientation in Guugu Yimithirr pointing gestures. Journal of Linguistic Anthropology 3(1). 3-45.

Haviland, John B. 2000. Pointing, gesture spaces, and mental maps. In McNeill, David (ed.), Language and gesture. 13-46. Cambridge: Cambridge University Press.

Haviland, John B. 2003. How to point in Zinacantán. In Sotaro Kita (ed.), Pointing: Where language, culture, and cognition meet. 147-178. Mahwah, NJ: Lawrence Erlbaum.

Healy, Christina. 2012. Pointing to show agreement. Semiotica 192. 175-195.

Holler, Judith, \& Steven C. Levinson. 2019. Multimodal language processing in human communication. Trends in Cognitive Sciences 23(8). 639-652.

Hou, Lynn \& Richard P. Meier. 2018. The morphology of first-person object forms of directional verbs in ASL. Glossa: A Journal of General Linguistics 3(1). 114. http://doi.org/10.5334/gjgl.469

Jarmołowicz-Nowikow, Ewa. 2015. How Poles indicate people and objects, and what they think of certain forms of pointing gestures. Lingua Posnaniensis 56(1). 85-95.

Johnston, Trevor. 2013a. Towards a comparative semiotics of pointing actions in signed and spoken languages. Gesture 13(2). 109-142.

Johnston, Trevor. 2013b. Formational and functional characteristics of pointing signs in a corpus of Auslan (Australian sign language): are the data sufficient to posit a grammatical class of 'pronouns' in Auslan? Corpus Linguistics and Linguistic Theory 9(1). 109 - 159.

Kendon, Adam. 1980. A description of a deaf-mute sign language from the Enga Province of Papua New Guinea with some comparative discussion. Part III: Aspects of utterance construction. Semiotica 32(3/4). 245-313.

Kendon, Adam. 2004. Gesture: Visible action as utterance. Cambridge: Cambridge University Press.

Kendon, Adam. 2010. Pointing and the problem of "gesture": Some reflections. Rivisti Di Psicolinguistica Applicata 10(3). 19-30.

Kita, Sotaro. 2003a. Pointing: A foundational building block of human communication. In Sotaro Kita (ed.). Pointing: Where language, culture, and cognition meet. 9-16. Mahwah, NJ: Lawrence Erlbaum. 
Kita, Sotaro. 2003b. Interplay of gaze, hand, torso orientation, and language in pointing. In Kita, Sotaro (ed.), Pointing: Where language, culture, and cognition meet. 307-328. Mahwah, NJ: Lawrence Erlbaum.

Kita, Sotaro., \& James Essegbey. 2001. Pointing left in Ghana: How a taboo on the use of the left hand influences gestural practice. Gesture 1(1). 73-95. http://doi.org/10.1075/gest.1.1.06kit

Klima, Edward. S. \& Ursula Bellugi. 1979. The signs of language. Cambridge, MA: Harvard University Press.

Koulidobrova, Elena \& Diane Lillo-Martin. 2016. A 'point' of inquiry: The case of the (non) pronominal IX in ASL. In Trutkowski, Eva, Helmut Weiss, Patrick Grosz \& Pritty Patel-Grosz (eds.), The impact of pronominal form on interpretation. 221-250.

Langacker, Ronald. W. 2008. Cognitive grammar: A basic introduction. New York: Oxford University Press.

Lee, Raymond L. \& Alistair B. Fraser. 2001. The rainbow bridge: Rainbows in art, myth, and science. University Park, PA: Penn State Press.

Le Guen, Olivier. 2006. L'organisation et l'apprentissage de l'espace chez les Mayas Yucatèques du Quintana Roo, Mexique. Paris: Université Paris X-Nanterre doctoral dissertation.

Le Guen, Olivier. 2011. Modes of pointing to existing spaces and the use of frames of reference. Gesture 11(3). 271-307. http://doi.org/10.1075/gest.11.3.021eg

Le Guen, Olivier. 2012. An exploration in the domain of time: from Yucatec Maya time gestures to Yucatec Maya Sign Language time signs. In Zeshan, Ulrike \& Connie de Vos (eds.), Endangered sign languages in village communities: Anthropological and linguistic insights. 209250. Berlin: Mouton de Gruyter \& Ishara Press.

Le Guen, Olivier \& Lorena Pool Balam. 2012. No Metaphorical Timeline in Gesture and Cognition Among Yucatec Mayas. Frontiers in Psychology 3. 1-15. http://doi.org/10.3389/fpsyg.2012.00271

Levelt, Willem J. M., Graham Richardson, \& Wido La Heij. 1985. Pointing and voicing in deictic expressions. Journal of Memory and Language 24. 133-164.

Levinson, Steven C. 2003. Space in language and cognition: Explorations in cognitive diversity 5. Cambridge University Press.

Levinson, Steven C. 2006. On the human “interaction engine.” In Enfield, Nicholas J \& Steven C. Levinson (eds.), Roots of human sociality: Culture, cognition and interaction. 39-69. Oxford: 
Berg.

Liddell, Scott K. 1996. Spatial representations in discourse: Comparing spoken and signed language. Lingua 98(1-3). 145-167.

Liddell, Scott K. 2000. Indicating verbs and pronouns: pointing away from agreement. In: Emmorey, Karen, \& Harlan Lane (eds.), The signs of language revisited: An anthology to honor Ursula Bellugi and Edward Klima. 303-320. Lawrence Erlbaum, Mahwah, NJ.

Liddell, Scott K. 2003. Grammar, gesture and meaning in American Sign Language. Cambridge: Cambridge University Press.

Liddell, Scott K. \& Melanie Metzger. 1998. Gesture in sign language discourse. Journal of Pragmatics 30(6). 657-697.

Lillo-Martin, Diane \& Edward S. Klima. 1990. Pointing out differences: ASL pronouns in syntactic theory. In Fischer, Susan D. \& Patricia Siple (eds.), Theoretical Issues in Sign Language Research 1. 191-210. University of Chicago Press.

MacLaughlin, Dawn. 1997. The structure of determiner phrases: Evidence from American Sign Language. Boston, MA: Boston University doctoral dissertation.

McBurney, Susan L. 2002. Pronominal reference in signed and spoken language: are grammatical categories modality-dependent? In: Meier, Richard P., Kearsy Cormier \& David Quinto-Pozos (eds.), Modality and structure in signed and spoken languages. 329--369. Cambridge: Cambridge University Press.

McBurney, Susan .L. 2004. Referential morphology in signed languages. Seattle, WA: University of Washington doctoral dissertation.

McClave, Evelyn C. 2007. Potential Cognitive Universals: Evidence from Head Movements in Turkana. In Duncan, Susan D., Justine Cassell \& Elena T. Levy (eds.), Gesture and the D dynamic dimension of language: Essays in honor of David McNeill.91-98. Philadelphia: John Benjamins.

McNeill, David. 1992. Hand and mind: What gestures reveal about thought. Chicago: Chicago University Press.

Meier, Richard P. 1990. Person deixis in American Sign Language. In: Fischer, Susan D., \& Patricia Siple (eds.), Theoretical issues in sign language research 1. 175-190. Chicago: Chicago University Press

Meier, Richard P. \& Diane Lillo-Martin. 2010. Does spatial make it special? On the grammar 
of pointing signs in American Sign Language. In Gerdts, Donna B, John Moore \& Maria Polinsky (eds.), Hypothesis A/hypothesis B: Linguistic explorations in honor of David M. Perlmutter. 345360. Cambridge, MA: MIT Press.

Meier, Richard. P \& Diane Lillo-Martin. 2013. The points of language. Humana.mente 24. 151-176.

Mesh, Kate. 2017. Points of comparison: What indicating gestures tell us about the origins of signs in San Juan Quiahije Chatino Sign Language. Austin, TX: The University of Texas at Austin doctoral dissertation.

Mesh, Kate \& Lynn Hou. 2018. Negation in San Juan Quiahije Chatino Sign Language: The integration and adaptation of conventional gestures. Gesture 17(3). 330-374. https://doi.org/10.1075/gest.18017.mes

Mesh, Kate. (Submitted). It's as far as the arm can raise: Pointing height marks target distance among the San Juan Quiahije Chatino.

Mesh, Kate, Emiliana Cruz, Joost van de Weijer, Niclas Burenhult \& Marianne Gullberg. 2021. Effects of scale on multimodal deixis: Evidence from Quiahije Chatino. Frontiers in Psychology. 11:584231. doi: 10.3389/fpsyg.2020.584231

Mihas, Elena. 2017. Interactional functions of lip funneling gestures: A case study of Northern Kampa Arawaks of Peru. Gesture 16(3). 432-479. https://doi.org/10.1075/gest.00004.mih

Morgenstern, Aliyah. 2014. Shared attention, gaze and pointing gestures in hearing and deaf children. In Arnon, Inbal, Marisa Casillas, Chigusa Kurumada, \& Bruno Estigarribia (eds), Language in interaction: Studies in honor of Eve V. Clark. 12. 139-156. Amsterdam: John Benjamins.

Müller, Cornelia. 2018. Gesture and sign: Cataclysmic break or dynamic relations? Frontiers in Psychology 9. 1651. https://doi.org/10.3389.fpsyg.2018.01651

Nonaka, Angela M. 2004. The forgotten endangered languages: Lessons on the importance of remembering from Thailand's Ban Khor Sign Language. Language in Society 33(5). 737-767.

Nonaka, Angela M. (2015). Toponyms in Ban Khor Sign Language. Learning Communities 16. 66--91.

Östling, Robert, Carl Börstell, \& Servane Courtaux. 2018. Visual iconicity across sign languages: Large-scale automated video analysis of iconic articulators and locations. Frontiers in Psychology 9. 725. 10.3389/fpsyg.2018.00725 
Padden, Carol. A. 1983. Interaction of morphology and syntax in American Sign Language. San Diego, CA: University of California, San Diego dissertation.

Peirce, Charles Sanders. 1940. Justus Buchler (ed.), Philosophical Writings of Peirce. New York: Dover.

Perniss, Pamela \& Asli Özyürek. 2015. Visible cohesion: A comparison of reference tracking in sign, speech, and co-speech gesture. Topics in Cognitive Science 7(1). 36-60.

Pfau, Roland. 2011. A point well taken. In Mathur, Gaurav \& Donna Jo Napoli (eds.), Deaf around the world: The impact of language. 144-163. New York: Oxford University Press.

Pfau, Roland \& Markus Steinbach. 2006. Modality-independent and modality-specific aspects of grammaticalization in sign languages. Linguistics in Potsdam 24. 5-98. Potsdam, Germany: Universitätsverlag Potsdam.

Piwek, Paul, Robert-Jan Beun, \& Anita Cremers. 2008. 'Proximal' and 'distal' in language and cognition: Evidence from deictic demonstratives in Dutch. Journal of Pragmatics 40(4). 694718. https://doi.org/10.1016/j.pragma.2007.05.001

Pyers, Jennie E. 2006. Indicating the body: Expression of body part terminology in American Sign Language. Language Sciences 28(2-3). 280-303.

Quine, Willard Van Orman. 1960. Word and object. Cambridge, MA: MIT Press

Schembri, Adam, Kearsy Cormier \& Jordon Fenlon. 2018. Indicating verbs as typologically unique constructions: Reconsidering verb 'agreement' in sign languages. Glossa: A Journal of General Linguistics 3(1). 89. http://doi.org/10.5334/gjgl.468

Schegloff, Emanuel. A. 1984. On some gestures' relation to talk. In J. Maxwell Atkinson, John Heritage \& Keith Oatley (eds.), Structures of social action. 266-298. Cambridge: Cambridge University Press.

Senghas, Anne \& Marie Coppola. 2001. Children creating language: How Nicaraguan Sign Language acquired a spatial grammar. Psychological Science 12(4). 323-328.

Shepard-Kegl, Judy A. 1985. Locative relations in American Sign Language word formation, syntax and discourse. Boston, MA: Massachusetts Institute of Technology dissertation.

Sherzer, Joel. 1973. Verbal and nonverbal deixis: The pointed lip gesture among the San Blas Cuna. Language in Society 2. 117-131.

Sherzer, Joel. 1983. Kuna ways of speaking: An ethnographic perspective. Austin, TX: University of Texas Press. 
Stukenbrock, Anja. (2014). Pointing to an 'empty' space: Deixis am Phantasma in face-toface interaction. Journal of Pragmatics 74. 70-93. http://doi.org/10.1016/j.pragma.2014.08.001

Talmy, Leonard. 2018. The targeting system of language. Cambridge, MA: MIT Press

Tomasello, Michael, Carpenter, Melinda, \& Ulf Liszkowski. (2007). A new look at infant pointing. Child development, 78(3), 705-722.

Torigoe, Takashi \& Wataru Takei. 2002. A descriptive analysis of pointing and oral movements in a home sign system. Sign Language Studies 2(3). 281-295.

van Hoek, Karen. 1992. Conceptual spaces and pronominal reference in American Sign Language. Nordic Journal of Linguistics 15(2). 183-199.

Wilbur, Ronnie B. 1999. Stress in ASL: Empirical evidence and linguistic issues. Language and

Speech 42(2-3). 229-250.

Wilcox, Sherman, \& Corrine Occhino. 2016. Constructing signs: Place as a symbolic structure in signed languages. Cognitive Linguistics 27(3). 371-404.

Wilkins, David. 2003. Why pointing with the index finger is not a universal (in sociocultural and semiotic terms). In Kita, Sotaro (ed.), Pointing: Where language, culture, and cognition meet. 171-215. Mahwah, NJ: Laurence Erlbaum.

Woodward, James. 1989. Basic color term lexicalization across sign languages. Sign Language Studies 63(1). 145-152.

Yano, Uiko, \& Kazumi Matsuoka (2018). Numerals and timelines of a shared sign language in Japan. Sign Language Studies 18(4), 640-665.

Zeshan, Ulrike \& Keiko Sagara (eds.). 2016. Semantic fields in sign languages: Colour, kinship and quantification 6. Walter de Gruyter.

Zimmer, June \& Cynthia G. Patschke. 1990. A class of determiners in ASL. In C. Lucas (ed.), Sign language research: Theoretical issues. 201-210. Washington, DC: Gallaudet University Press. 